In: Hans-Joachim Veen (Hg.): Das Bild der DDR in Literatur, Film und Internet, Köln/Weimar/Wien, Böhlau Verlag, 2015, S. 81-100.

Matthias Steinle

\title{
Drei Krisen und das Wunder ihres Endes: Die DDR im deutschen Dokudrama
}

Man stelle sich vor, es ist ein Jubiläumsjahr des Mauerfalls und nicht nur das spektakulärste, sondern auch noch das einzige Dokudrama zum Thema DDR überhaupt läuft auf einem Kulturkanal... ${ }^{1}$ Unvorstellbar im deutschen Fernsehen nach der Jahrtausendwende, das geprägt war von historischen Eventfilmen, die die deutsche Geschichte nach den Regeln der Jahrestagsdramaturgie massenmedial begleitet und mitgeschrieben haben. Unvorstellbar und doch so geschehen im Jahr 2014, in dem Arte mit ZugindieFreiheit (Sebastian Dehnhard/Matthias Schmidt) das einzige Dokudrama zum 25. Jahrestag der dramatischen Ereignisse in der DDR ausgestrahlt hat. ${ }^{2}$ Der Film zeigt die spannungreiche Reise der Prager Botschaftsflüchtlinge mit dem Zug über das Territorium der DDR in die Bundesrepublik im Sommer '89 in der für das Format charakteristischen Mischung aus Zeitzeugeninterviews, Spielszenen und Archivbildern. Dass es sich im Erinnerungsjahr 2014 um das einzige aufwändige Dokudrama zum Thema DDR und deren Ende überhaupt handelte, ließe sich auch als Indiz dafür lesen, dass dokufiktionale Genreformen des Eventfernsehens ihre Dominanz ebenso eingebüßt haben wie die mit ihnen transportierten erinnerungskulturellen Narrative, die im Folgenden analysiert werden sollen.

Filme zur deutsch-deutschen Geschichte trafen nach der Vereinigung auf zwei unterschiedliche, von der Teilung geprägte Erinnerungskulturen: eine westdeutsche, die unter dem Vorzeichen der Kontinuität die Geschichte der alten Bundesrepublik weitgehend

\footnotetext{
${ }^{1}$ Bei dem Artikel handelt es sich um eine gekürzte und aktualisierte Fassung von Matthias Steinle: Good Bye Lenin - Welcome Crisis! Die DDR im Dokudrama des historischen Event-Fernsehens, in: Tobias Ebbrecht/Hilde Hoffmann/Jörg Schweinitz (Hg.): DDR erinnern, vergessen. Das visuelle Gedächtnis des Dokumentarfilms, Marburg 2009, S. 322-342.

${ }^{2}$ Die MDR-Arte-Produktion Zug in die Freiheit lief auf Arte um 20.15 Uhr, die ARD zeigte den Film darauf am 3. Oktober um 18.30 Uhr und der MDR am 2. November um 20.15 Uhr.
} 
konsensuell als success story einer »geglückte[n] Demokratie « ${ }^{3}$ schrieb; und eine ostdeutsche, die von der Erfahrung eines radikalen Bruchs geprägt war, wobei es zu zentralen Fragen der Einordnung und Bewertung der DDR kein allgemeines Selbstverständnis gab. Für die ehemaligen DDR-Bürger ging es immer auch »um die Auf- oder Abwertung gelebten Lebens $\aleph^{4}$. Das Fernsehen als zentrale gesellschaftliche Agentur der Erinnerungsarbeit stand vor der Aufgabe, ein gesamtdeutsches und zugleich erinnerungskulturell gespaltenes Publikum anzusprechen. Welche DDR-Geschichtsbilder und -deutungen vor diesem Hintergrund angeboten wurden, soll im Folgenden am Beispiel von publikumswirksamen Dokudramen erörtert werden, die, nicht zuletzt aufgrund ihres ökonomischen Aufwandes, ein möglichst großes Publikum beiderseits der Elbe ansprechen mussten. ${ }^{5}$

\section{Zeitgeschichte im deutschen Dokudrama}

Die im neuen Jahrtausend im Rahmen der televisuellen Eventgeschichtskultur in Deutschland produzierten Dokudramen lassen sich durch eine Reihe thematischer, ästhetischer und ökonomischer Gemeinsamkeiten charakterisieren: Generell behandeln diese zeitgeschichtliche Stoffe ab 1933, die am Beispiel individueller Geschichten durch Personalisierung, Emotionalisierung und Dramatisierung vermittelt werden. ${ }^{6}$ Verschiedene dokumentarisierende Strategien (Betonung einer wahren Geschichte, genaue Zeit- und Ortsangaben, Identifizierung der Akteure/Zeitzeugen, historische Kulissen, Archivmaterial, Mitarbeit von Historikern) sollen Authentizität evozieren und die gesellschaftliche Relevanz des Themas betonen, die durch »extra-textuelle Ereignisse ${ }^{7}$ wie produktionsbegleitende Titelstories, Talkshows und Making-ofs vermittelt wird. Dabei dominiert ein enges Geflecht

\footnotetext{
${ }^{3}$ Edgar Wolfrum: Die geglückte Demokratie. Geschichte der Bundesrepublik Deutschland von ihren Anfängen bis zur Gegenwart, Stuttgart 2006.

${ }^{4}$ Annette Leo: Keine gemeinsame Erinnerung. Geschichtsbewusstsein in Ost und West, in: Aus Politik und Zeitgeschichte, Jg. 53 (2003), Bd. 40-41, S. 27-32, hier S. 27.

${ }^{5}$ Entsprechend der Funktion als »historisches Ereignisfernsehen« (Tobias Ebbrecht) werden im Folgenden unter Dokudrama pragmatisch sowohl Filme verstanden, in denen der dokumentarische Aspekt stärker betont wird, wie in den Produktionen von Guido Knopp/Hans-Christoph Blumenberg, als auch Filme, die am HollywoodHistorienspektakel orientiert sind, wie die von teamWorx als »tv Event« vermarkteten Filme. Vgl. Tobias Ebbrecht/Matthias Steinle: Dokudrama in Deutschland als historisches Ereignisfernsehen - eine Annährung aus pragmatischer Perspektive, in: MEDIENwissenschaft, Nr. 3 (2008), S. 250-255.

${ }^{6}$ In der extremen Konzentration auf Zeitgeschichte unterscheiden sich die deutschen Dokudramen etwa von den ästhetisch und in ihrer soziokulturellen Funktion ähnlich gelagerten französischen »fictions patrimoniales «, die thematisch bis in die frühe Neuzeit zurückgehen. Vgl. Pierre Beylot/RaphaëlleMoine (Hg.): Les fictions patrimonialessur grand et petit écran. Contours et enjeux d'un genre intermédiatique, Bordeaux 2009.

${ }^{7}$ Derek Paget: No Other Way to Tell It. Dramadoc/Docudrama on Television, Manchester/New York 1998, S. 80 .
} 
der beteiligten Akteure (Schauspieler, Regisseure, Produzenten) im Modus des Star-Systems. Thematisch lassen sich die Produktionen des historischen Ereignisfernsehens grob drei Bereichen zuordnen: 1. »Drittes Reich «, 2. westdeutsche Nachkriegsgeschichte/BRD und 3. DDR.

Charakteristisch für den personalisierten und auf das »Schicksal« der Deutschen reduzierten Zugriff auf den Komplex »Drittes Reich« ist die Entlastungstendenz, die Verantwortung auf wenige Funktionsträger abzuschieben und die deutsche Bevölkerung primär als Opfer des Nationalsozialismus $\mathrm{zu}$ zeichnen. ${ }^{8}$ So inszenieren jüngere deutsche Melodramen, die Judenverfolgung und -vernichtung thematisieren, rückwirkend positive deutsch-jüdische Identitätsstiftung und spielen im unreflektiert-nostalgischen Ufa-Stil »Versöhnungsmelodien ${ }^{9}$.

Im Mittelpunkt der Filme über die westdeutsche Geschichte stehen gemeinschaftsstiftende Erfahrungen, die wie in DasWundervonBern (Sönke Wortmann, 2003) der Bundesrepublik nationale Identität verleihen. Eine Bedrohung erfolgt generell von außen wie die Blockade in DieLuftbrücke (Dror Zahavi, 2005). Krisen resultieren aus Unfällen, so wie etwa das Grubenunglück in DasWundervonLengede(Kaspar Heidelbach, 2003), oder aus Naturkatastrophen wie der Hamburger Sturmflut von 1962 in DieSturmflut (Jorgo Papavassilou, 2005). All diese Krisen werden kollektiv bewältigt. Obwohl solche am Katastrophenfilm orientierten Dokudramen sich scheinbar weniger für politische Implikationen, sondern vielmehr für dramatische Effekte, nostalgische Bilder, Familien- oder Liebesgeschichten mit visuellen Schauwerten interessieren, bedienen sie das Bedürfnis nach dem Austritt aus der Geschichte als politisch konfliktuellem Feld und nach nationaler Mythenbildung. »Jedes Volk braucht eine Legende«, verkünden entsprechend programmatisch Trailer und Filmplakat zu DasWundervonBern. Dokudramen mit kritischen Tönen zur westdeutschen Geschichte erreichen nur selten Eventcharakter und wenn, dann greifen andere mediale Mechanismen wie beispielsweise im Fall von Contergan (Adolf

\footnotetext{
${ }^{8}$ Vgl. Tobias Ebbrecht: Geschichtsbilder im medialen Gedächtnis. Filmische Narrationen des Holocaust, Bielefeld 2011.

9 Sonja M. Schultz: Die harmonische Leinwand. Filmische Stereotypen bei der Darstellung von Nationalsozialismus und Holocaust in aktuellen deutschen Produktionen, in: apropos: Film 2005, Berlin 2005, S. 76-88, hier S. 88.
} 
Winkelmann, 2005). ${ }^{10}$ Die einzige thematische Ausnahme stellt der Komplex »Terrorismus « mit der RAF als aus der eigenen Gesellschaft erwachsenen Bedrohung dar. Bezeichnenderweise endet Breloers wegbereitender Zweiteiler Todesspiel (1997) über die Schleyer- und Landshut-Entführung unmittelbar nach der akuten Krisensituation. Die teamWorx-Produktion Mogadischu (Roland S. Richter, 2008) konzentriert sich ausschließlich auf die Flugzeugentführung. Widersprüche, nach wie vor offene Fragen und vor allem die Konsequenzen des »Deutschen Herbstes«, das langfristig vergiftete politische Klima, geraten durch die Beschränkung auf den Krisenmoment nicht in den Blick. ${ }^{11}$

Im Kontrast zur mythologisierenden Geschichtsschreibung der Bundesrepublik im historischen Ereignisfernsehen steht als dritter thematischer Komplex die Auseinandersetzung mit der DDR. Die Wahrnehmung des »anderen« deutschen Staates erfolgt ausschließlich unter den Vorzeichen krisenhafter Ereignisse anhand der drei Fixpunkte: 17. Juni 1953, 13. August 1961 und 9. November 1989, mit dem zugleich die DDR in den bundesdeutschen »Wunder-Diskurs « überführt wird.

Zum fünfzigsten Jahrestag 2003 wurde dem Aufstand vom 17. Juni mit einer so außergewöhnlichen Intensität gedacht, dass vom »medialen Overkill « ${ }^{12}$ die Rede war. HansChristoph Blumenberg rekonstruierte unter der Redaktion von Guido Knopp die Ereignisse in der Berliner Stalinallee in DerAufstand(2003), für den ausgiebig Zeitzeugen befragt wurden. Die Sendeanstalten der ARD machten sich untereinander Konkurrenz mit ZweiTageHoffnung (Peter Keglevic, 2003) und TagedesSturms (Thomas Freundner, 2003). Thematisch allerdings ergänzten sich die beiden Filme durch den Fokus des Erstgenannten auf das »Epizentrum des Bebens « in Berlin und des Letztgenannten auf die Auswirkungen in der Bitterfelder Provinz.

Den 13. August 1961 riefen die beiden teamWorx-Produktionen DerTunnel (Roland S. Richter, 2001) sowie DieMauer-Berlin '61 (Hartmut Schoen, 2005) ins Bewusstsein. Zum zwanzigsten Jahrestag des Mauerfalls erinnerte die Sat.1-Produktion BöseckendorfDieNacht,indereinDorfverschwand (Oliver Dommenget, 2009) an die Massenflucht einer

\footnotetext{
${ }^{10}$ Dass die Ausstrahlung des Zweiteilers über den Pharmaskandal im November 2007 zum Ereignis wurde, lag vor allem an dem von der Berichterstattung ausführlich begleiteten zweijährigen Rechtsstreit um die Mischung aus Fakt und Fiktion.

${ }^{11}$ Vgl. Kay Hoffmann: Zehn Jahre nach Breloers »Todesspiel«, in: Rundfunk und Geschichte, Jg. 33 (2007), H. 3-4, S. 71-73, online abrufbar unter: http://rundfunkundgeschichte.de/assets/RuG_2007_3-41.pdf, letzter Zugriff: 16.03.2015.

${ }^{12}$ Marc-Dietrich Ohse: Rückblicke, Aussichten. Erinnerungskultur und deutsche Befindlichkeit, in: Deutschland Archiv, Jg. 36 (2003), H. 6, S. 924-928, hier S. 925.
} 
Dorfgemeinschaft im Oktober 1961 an der innerdeutschen Grenze. Zum fünfzigsten Jahrestag des Mauerbaus widmete sich die Arte-Produktion Geheimsache Mauer - Die Geschichte einer deutschen Grenze (Christoph Weinert/Jürgen Ast, 2011) der Berliner Mauer und befragte dazu auch jene, die sie bewacht haben.

Die letzte Etappe der DDR zeigt der Zweiteiler Deutschlandspiel (2000) von Blumenbergund Knopp unter Aufbietung zahlreicher prominenter Politiker, der mit einer kriselnden DDR an ihrem vierzigsten Jahrestag einsetzt und mit dem Vereinigungsfeuerwerk am 3. Oktober 1990 endet. Danach fand das Thema Mauerfall erst mit dem Nahen des zwanzigjährigen Jubiläums wieder Eingang in die Produktionspläne: So der im Auftrag von RTL 2007 gedrehte Film Prager Botschaft (Lutz Konermann), der mit 1.800 Komparsen an Originalschauplätzen in Prag die dramatische Situation in der bundesdeutschen Botschaft im September'89 nachstellte. Wenn die DDR im deutschen Dokudrama ein Wunder erleben darf, dann das ihres Endes: Entsprechend realisierte im gleichen Jahr teamWorx-Produzent Nico Hofmann für das ZDF Das Wunder von Berlin (Roland S. Richter, 2007), eine Familiengeschichte aus der Endphase der DDR, die mit dem Mauerfall endet. ${ }^{13}$ Der MDR lieferte die Ereignisse aus der Provinz mit Das Wunder von Leipzig - Wir sind das Volk (Sebastian Dehnhardt/Matthias Schmidt, 2009). Die Konkurrenz um die Aufmerksamkeit der Fernsehzuschauer führte dazu, dass der erste Film zum Erinnerungsjahr 2009 bereits 2008 ausgestrahlt wurde: Die Sat.1Produktion Wir sind das Volk - Liebe kennt keine Grenzen (Thomas Berger, 2008) beginnt mit einem gescheiterten Fluchtversuch über die deutsch-deutsche Grenze und endet mit dem Mauerfall als Happyend.

Die DDR steht in den deutschen Nachwendedokudramen nur als Ruine auf, deren Verfall anhand von Krisendaten erzählt wird, wie dies beispielhaft die ZDF-Produktion Die Wölfe (Friedemann Fromm, 2009) demonstriert: Der Dreiteiler zeigt die Schicksale der Mitglieder der Jugendbande »Die Wölfe« anhand der Ereignisse um die Berlin-Blockade, den Mauerbau und den Mauerfall. Die Wahrnehmung der DDR unter krisenhaften Vorzeichen schreibt sich auch in die Zukunft fort wie in dem teamWorx-Zweiteiler Die Grenze (Roland S. Richter, 2010), der im Jahr 2010 spielt: In einer sich verschärfenden wirtschaftlichen und sozialen Krise spaltet sich Mecklenburg-Vorpommern vom Rest der Republik ab und bildet eine $» k l e i n e \mathrm{DDR} \ll$.

\footnotetext{
${ }^{13}$ Zum »Etikettenschwindel « des Filmtitels und dem Bedürfnis nach Wundern im deutschen Film siehe Matthias Dell: Wunder gibt es immer wieder. Warum wird alles, was dieses Land als Höhepunkt erlebt, ins Märchenhafte gesteigert?, in: Der Freitagvom 25. Januar 2008.
} 
Die wenigen Dokudramen, die nicht an den krisenbesetzten Schlüsseldaten ansetzen, orientieren sich an den damit verbundenen Erinnerungsorten, so etwa Die Frau vom Checkpoint Charlie (Annette Hess/Miguel Alexandre, 2007). Der ARD-Zweiteiler, der in den 1980er Jahren spielt, zeigt den Kampf einer von Veronica Ferres verkörperten (Super-) Mutter nach ihrem Freikauf durch die Bundesrepublik um ihre in der DDR verbliebenen Kinder. Daneben interessieren sich die Dokudramen noch für die Grenze wie in dem an der Biographie von Michael Gartenschläger orientierten Film Die Todesautomatik (Niki Stein, 2007).

\section{Zur Rolle von Genremustern im »Untergang auf Raten«}

In der Konstruktion der DDR als permanente Krise greifen genrespezifische, geschichtspolitische und erinnerungskulturelle Aspekte wirksam ineinander. Krisenhafte Situationen bieten sich für die Dramaturgie von Dokudramen an: Einer in sich gespaltenen Welt, wie sie das geteilte Deutschland prototypisch repräsentiert, steht die für das klassische Drama typische Einheit der Handlung gegenüber. Strenge Kausalität und überzeugende Motivierung produzieren eine eindeutige Wirkung, auf die das Dokudrama, verstanden als persuasive Praxis, abzielt. Hinzu kommt bei Dokudramen die häufige Dominanz des Melodramatischen, das dezidiert moralische Urteile vermittelt: Indem das Filmmelodrama häusliche Milieus und Familienbilder in den Kontext eines größeren sozialen Systems stellt, das die Erzählung als mächtig und korrupt enthüllt, wird eine klare moralische Perspektive entwickelt. ${ }^{14}$ Dieses Genremuster bietet sich für eine dokudramatische Narrativisierung der DDR an, war diese doch mit Abriegelung ihrer Grenzen verantwortlich für das Auseinanderreißen von Familien. Entsprechend exemplarisch lassen sich private Bereiche und Familienbilder als Zeichen menschlicher Verbundenheit und - deutscher - Einheit der SEDMachtpolitik als Inbegriff von Unterdrückung und Spaltung Deutschlands gegenüberstellen. Gemäß dem polarisierenden Genreschema einer klaren Trennung in Gut und Böse werden emotionale Schwarzweißbilder vermittelt, die im Kontext der deutschen Teilung auf den Feindbildern des Kalten Krieges aufbauen können. Der Rekurs auf etablierte Klischees aus Zeiten der Systemkonkurrenz bietet sich auch aus Gründen der Erzählökonomie an, da damit

\footnotetext{
${ }^{14}$ Vgl. Steve Lipkin: Defining Docudrama: In the Name of the Father, Schindler's List, and JFK, in: Alan Rosenthal (Hg.): Why Docudrama? Fact-Fiction on Film and TV, Carbondale/Edwardsville 1999, S. 370-383, hier S. $372 \mathrm{f}$.
} 
ein Repertoire an Motiven und Darstellungsstrategien bereitsteht, das für Eindeutigkeit sorgt und an langfristige mediale Erfahrungen anschließen kann. Indem »Zonen der Sicherheit und Bestimmbarkeit ${ }^{15}$ geschaffen werden, kommt auch die Trostfunktion des Genres zum Tragen. Nicht zuletzt verweist die Häufigkeit der Thematisierung auf nationale Traumata. Wie im Melodram vermittelt auch das Dokudrama die Hoffnung, verlorene moralische Kategorien könnten zurückgewonnen und erneuert werden. ${ }^{16}$ Dieses Genremuster dient als Modell für die Geschichte von der friedlichen Überwindung der DDR und der Wiedervereinigung.

Die unterschiedliche thematische Behandlung von Bundesrepublik und DDR reflektiert erinnerungskulturelle Bedürfnisse ebenso wie geschichtspolitische Interessen. In der Retrospektive aktueller Dokudramen darf die DDR kein Ort gemeinschaftsstiftender Ereignisse sein. Die Konzentration auf die drei Krisendaten als einzig signifikante Erinnerungsorte für die DDR vermittelt die teleologische These vom »Untergang auf Raten ${ }^{17}{ }^{17}$. Auf der Strecke bleibt dabei die Interaktion von Herrschaft und Gesellschaft, die nach Schließung der DDR-Grenzen die relative Stabilität bis in die 1970er Jahre erklärt. ${ }^{18}$ Verdrängt wird damit auch die Komplexität deutsch-deutscher Wahrnehmung in ihrer historischen Dimension: Gab es doch in der Bundesrepublik neben den Feindbildern des Kalten Krieges lange Zeit eine breite Palette von Fremd-, aber auch von öffentlich-rechtlich produzierten Freundbildern. ${ }^{19}$ Diese stellen für die Verantwortlichen aus der Bundesrepublik eine unliebsame Erinnerung dar und sind dementsprechend ein weitgehend blinder Fleck im öffentlichen Erinnern.

Im Ensemble der Produktionen ist die Themenwahl symptomatisch für mediale Wahrnehmungsraster der Zeitgeschichte, die sich zugespitzt folgendermaßen zusammenfassen lassen: das »Dritte Reich«als große Leidensgeschichte aller Deutschen, die DDR als krisengeschütteltes »Phänomen« (Kurt Georg Kiesinger) mit der Bevölkerung

\footnotetext{
${ }^{15}$ Thomas Waitz: Geschehen/Geschichte. Das Dokudrama bei Hans-Christoph Blumenberg, in: Harro Segeberg (Hg.): Referenzen. Zur Theorie und Geschichte des Realen in den Medien, Marburg 2009, S. 211-222, hier S. 219.

${ }^{16}$ Vgl. Lipkin: Defining Docudrama, a. a. O., S. 373.

${ }^{17}$ Armin Mitter/Stefan Wolle: Untergang auf Raten. Unbekannte Kapitel der DDR-Geschichte, München 1993.

${ }^{18}$ Vgl. »Die Empfehlungen der Expertenkommission zur Schaffung eines Geschichtsverbundes >Aufarbeitung der SED-Diktatur««, in: Martin Sabrow u. a. (Hg.): Wohin treibt die DDR-Erinnerung? Dokumentation einer Debatte, Bonn 2006, S. 17-43, hier S. 34 f.

${ }^{19}$ Vgl. Matthias Steinle: Vom Feindbild zum Fremdbild. Die gegenseitige Darstellung von BRD und DDR im Dokumentarfilm, Konstanz 2003, S. 333 ff.
} 
beiderseits der Grenze/Mauer als Opfer und die Bundesrepublik als solidarische Gemeinschaft und wundersame Erlösung aus der Leidensgeschichte.

\section{Westdeutsche Redefinition deutscher Erinnerungsorte}

Die DDR als Fernsehereignis wird im Wesentlichen von wenigen Regisseuren, Redakteuren und Produzenten auf den Bildschirm gebracht, die im Allgemeinen aus Westdeutschland stammen. Im zeitzeugengestützten Dokudrama ist es vor allem der ehemalige Filmkritiker Hans-Christoph Blumenberg, der sich für das ZDF unter der Redaktion von Guido Knopp und produziert von Ulrich Lenze (Cinecentrum) der Geschichte angenommen hat. Die fiktional orientierten »Event-Movies« dominieren die Filme der von Nico Hofmann geleiteten Produktionsfirma teamWorx. Als Tochter der Ufa-Gruppe steht der Name für international marktfähige Zeitgeschichte auf der Höhe computeranimierter Spezialeffekte. ${ }^{20}$ Die Regisseure der teamWorx-Produktionen sind alle westlich sozialisiert wie die Darsteller der Hauptrollen. Bevorzugt werden aus Gründen internationaler Marktfähigkeit »eingeführte Marken ${ }^{21}$, allen voran Heino Ferch, gefolgt von Sebastian Koch, Veronica Ferres und Alexandra Maria Lara. Deren Performanz hat für ehemalige DDR-Bürger häufig einen Verfremdungseffekt - wenn auch nicht im Brecht'schen Sinn - zur Folge. Jochen Schmidt veranlasst eine Szene in Die Frau vom Checkpoint Charlie, in der Veronica Ferres als »Mutter Courage der Republikflucht« im weißen Hemd eine Kohlenschütte unterm Arm trägt, zu folgender Reflexion:

Es könnte einmal eine Art Königsdisziplin für Schauspieler aus dem Westen werden, einen authentischen Ossi darzustellen, wie amerikanische Schauspieler ja immer Minderwertigkeitskomplexe haben, weil sie Shakespeare mit ihrem Akzent nie richtig spielen können. ${ }^{22}$

Das Filmerbe der DDR ist personell nur in Nebenrollen präsent. Visuell ist es in fast allen Filmen in Form dokumentarischer Aufnahmen der DEFA-Wochenschau Der Augenzeuge und des DDR-Fernsehens als Quellenmaterial zu sehen, das entweder illustrative oder dramaturgisch-binnendiegetische Funktion hat. Eigenständige ästhetische Qualitäten oder ein originär dokumentarisches Interesse wird den DDR-Bildern durch diesen Umgang abgesprochen.

\footnotetext{
${ }^{20}$ Vgl. Nico Hofmann: Exzellenz-Wettbewerb. Ein epd-Interview mit teamWorx-Produzent Nico Hofmann, in: epd medien, Nr. 60, vom 1. August 2007, S. 3-6.

21 Eike Wenzel: Retrotopia oder Erinnerungsland, in: Burkhard Röwekamp/Matthias Steinle (Hg.): Selbst/Reflexionen. Von der Leinwand bis zum Interface, Marburg 2004, S. 64-78, hier S. 76.

${ }^{22}$ Jochen Schmidt: Die DDR im Film. Wie mutig wir waren!, in: Frankfurter Allgemeine Sonntagszeitung vom 23. September 2007, S. 35.
} 
Zunächst sorgen authentische Aufnahmen für Zeitkolorit; solche Schlüsselbilder sollen beim Zuschauer entsprechende Assoziationen hervorrufen und werden daher häufig im Vorspann oder in der Exposition eingesetzt. Zwei Tage Hoffnung beispielsweise unterlegt den eingangs zu hörenden Kommentar eines fiktiven RIAS-Sprechers, der von Fluchten aus der DDR berichtet, mit dokumentarischen Aufnahmen aus Berlin und überfüllten Notaufnahmelagern. Programmatisch verschränkt der Film so die Ebenen von fact und fiction.

Der Vorspann von Der Tunnel zeigt in einem Binnenkader bekannte Bilder vom Mauerbau, auf der Tonebene begleitet von Originaltönen des DDR-Rundfunks, womit die Ereignisse des 13. August erzählökonomisch etabliert sind (Abb. 1).

\section{Abb. 1 Archivbilder einer Ulbricht-Rede im Vorspann zu Der Tunnel}

Im Folgenden werden in Der Tunnel keine Archivbilder mehr mit dokumentarischem Gestus verwendet; zwar werden noch häufiger Ausschnitte aus DEFA-Wochenschauen gezeigt, diese sind aber als binnendiegetische Elemente der Spielhandlung auf der Leinwand von Kinos zu sehen, die als konspirative Treffpunkte in Ostberlin dienen. Die Auswahl der so als Film-imFilm gezeigten Dokumentaraufnahmen, zynische Mauerrechtfertigungsreden von SEDFunktionären und marschierende Betriebskampfgruppen, tragen zur historisierenden Rahmung der Spielhandlung bei und legitimieren das Tunnelbauprojekt moralisch.

Ein wesentliches Element der Filme sind »sekundäre Anschauungsbilder«, das heißt indirekte Bildzitate, die historischen Filmaufnahmen nachempfunden sind. Die Mauer - Berlin '61 zeigt, wie Pfähle in den Boden gelassen werden, um daran Stacheldraht zu befestigen. Dabei handelt es sich um eine detailgetreue Rekonstruktion dokumentarischer Bilder vom Mauerbau. Das Spiel mit Zitaten und Gegenzitaten, die als »Echo-Kino ${ }^{23}$ auf frühere Darstellungen verweisen, ohne dass deren Herkunft erkennbar wird, ist ein Charakteristikum der historisierenden Dokudramen. Der Tunnel betreibt »Echo-Kino« mit der postmodernen Attitüde des Pastiche, wie der Umgang mit der visuellen Ikone des Mauerbaus verdeutlicht: Die Wochenschau-Aufnahmen vom fliehenden NVA-Soldaten Conrad Schumann, der in der Bernauer Straße beim Sprung über den Stacheldraht sein Gewehr wegwirft (Abb. 2), sind

\footnotetext{
${ }^{23}$ Sylvie Lindeperg: Spuren, Dokumente, Monumente. Filmische Verwendung von Geschichte. Historische Verwendung des Films, in: Eva Hohenberger/Judith Keilbach (Hg.): Die Gegenwart der Vergangenheit. Dokumentarfilm, Fernsehen und Geschichte, Berlin 2003, S. 65-81, hier S. 68.
} 
detailgetreu nachinszeniert worden, nur dass sich die Szene in Der Tunnel vor dem Brandenburger Tor ereignet (Abb. 3-5).

Abb. 2 Historische Aufnahme der Flucht des NVA-Soldaten Conrad Schumann in der Bernauer Straße am 15. August 1961

Abb. 3-5 Nachinszenierung der Flucht des eines NVA-Soldaten am Filmanfang von Der Tunnel

Zur Schauwertsteigerung werden $\gg$ Superzeichen ${ }^{24}$ der Teilungsgeschichte verdichtet, was auch deren Erinnerungsfunktion affiziert: Das Verschmelzen vom Bild des flüchtenden NVASoldaten als westdeutsche Ikone des Mauerbaus mit dem Brandenburger Tor als Wahrzeichen der deutschen Geschichte schlechthin stellt den gesamtdeutschen Erinnerungsort »Brandenburger Tor« unter die Vorzeichen westdeutscher Erinnerungskultur.

Neben der Verdichtung von Schlüsselbildern besteht ein weiteres Verfahren zur Schaffung sekundärer Anschauungsbilder in der Selbstinszenierung des Films als Quelle: Wie bereits erwähnt, verzichtet Der Tunnel mit Ausnahme des Vorspanns auf den nichtdiegetischen Einsatz von Dokumentarmaterial. Stattdessen schafft er solches selbst: Der historische Tunnelbau wurde von einem Filmteam des amerikanischen Fernsehsenders NBC begleitet, das hier in die Spielhandlung integriert wird. Mehrfach sind mit der Handkamera gedrehte, grobkörnige Schwarzweißbilder von den Grabungsarbeiten eingeschnitten, nur dass nicht dokumentarische Aufnahmen aus den 1960er Jahren zu sehen sind, sondern in Großaufnahme gut erkennbar die Gesichter der Schauspieler des Jahres 2001 (Abb. 6). Der nachgestellte Charakter dieser »sekundären Anschauungsbilder« ist eindeutig zu erkennen und zeitigt widersprüchliche, medienreflexiv-aufklärerische und selbstlegitimatorisch-illusionierende Effekte: Einerseits gelingt dem Film so der Spagat, den fiktionsstörenden Blick in die Kamera diegetisch einzubinden und gleichzeitig im Verweis auf das historische Material den fiktionalen Charakter der Bilder auszustellen. So wird nicht nur die Historizität der Vorlagen deutlich; die demonstrative Aneignung in Form sekundärer Anschauungsbilder führt auch die mediale Verfasstheit der Geschichte vor Augen und verweist auf die Prägekraft der Medien in der Wahrnehmung des Historischen. Andererseits inszeniert sich der Film damit selbst als

\footnotetext{
${ }^{24}$ Manuel Köppen: Von Effekten des Authentischen - Schindlers Liste: Film und Holocaust, in: Ders./Klaus R. Scherpe (Hg.): Bilder des Holocaust. Literatur - Film - Bildende Kunst, Köln/Weimar/Wien 1997, S. 145-170, hier S. 146.
} 
Quelle; das filmische Zeichen verweist nicht mehr »auf seine Referenz, sondern verwandelt sich selbst in den Referenten, der in der Geschichte sein bloßes Zeichen sucht $\ll^{25}$.

Abb. 6 Mit der Handkamera gedrehtes Schwarzweißbild der Schauspielerin Nicolette KrebitzErreur! Signet non défini. in Der Tunnel, das den Bildern der NBC-Reportage The Tunnel (1962) nachempfunden ist

Im Gegensatz zu den teamWorx-Produktionen arbeitet Hans-Christoph Blumenbergentsprechend der von Heinrich Breloer etablierten Technik ausgiebig mit historischem Bildmaterial. Dessen Herkunft und seine vorgängige ästhetische und ideologische Diskursivierung werden in Der Aufstand nur ein einziges Mal ansatzweise thematisiert: Bevor die Unruhen in der Stalin-Allee beginnen, zeigt der Film bunte Bilder eines lichten Alltags in Ostberlin, die eine Sprecherstimme aus dem Off kommentiert: »Diese Propagandabilder täuschen, die Bevölkerung leidet um so mehr unter staatlichem Zwang.« Letzten Endes dienen die DDR-Selbstbilder nur als Stichwortgeber eines allwissenden Kommentars. In Deutschlandspiel werden die heimlichen Aktivitäten von Video-Journalisten bei den Montagsdemonstrationen in Spielszenen gezeigt, woraufhin die entsprechenden dokumentarischen Bilder eingeblendet werden. Fragen, was mit den Bildern geschah und wie diese von wem und wann genutzt wurden, werden nicht gestellt.

Die Archivbilder haben Evidenzcharakter und werden im Knoppschen Stil instrumentalisiert, das heißt in Form kurzer Einstellungen mit illustrativem und/oder assoziativem Charakter affirmativ im Sinne dokumentarischer Selbstevidenz verwendet. Es kommt weniger auf den dokumentarischen Wert der Bilder an als vielmehr auf den Wert der durch die Bilder hervorgerufenen Erinnerungsleistung. So kommen die Archivbilder im ZDF-Dokudrama zu ihrem Ausdruck als Monument, nicht aber zu ihrem Recht als Dokument. ${ }^{26}$

In Blumenbergs Aufstandwerden die Demonstrationen vom 17. Juni im Wechsel von Zeitzeugenaussagen, Archivbildern und rekonstruierten Szenen dargestellt, die durch ihren wechselseitigen Bezug zu einer selbstreferentiellen Einheit verschmelzen: Das Archivmaterial zeigt, wovon die Zeitzeugen berichten, und die Spielszenen stellen zum einen die Archivbilder nach und setzen zum anderen die Zeitzeugenerinnerung in Szene. Zum Mythos des Archivbildes als scheinbar unverstelltem Zugang zur Vergangenheit gesellt sich der

\footnotetext{
${ }^{25}$ Michael Wildt: Der Untergang: Ein Film inszeniert sich als Quelle, in: Zeithistorische Forschungen/Studies in Contemporary History, Online-Ausgabe 2 (2005), H. 1, http://www.zeithistorische-forschungen.de/12005/id=4760, letzter Zugriff: 17.01.2015.

${ }^{26}$ Vgl. Matthias Steinle: Das Archivbild und seine Geburt als Wahrnehmungsphänomen in den 1950er Jahren, in: Corinna Müller/Irina Scheidgen (Hg.): Mediale Ordnungen. Erzählen, Archivieren, Beschreiben, Marburg 2007, S. 241-264, hier S. 262.
} 
Gleiches versprechende Mythos des Zeitzeugen. Die durch den Hybridcharakter produzierten Brüche verschmilzt die Montage zu einer glatten Oberfläche realgeschichtlicher Evidenz, die »Historie scheint sich selbst zu erzählen. $\ll^{27}$ Besonders problematisch ist Blumenbergs Arbeit mit $»$ sekundären Erinnerungsbildern $\ll^{28}$, das heißt die szenische Gestaltung der Aussagen von Zeitzeugen, für die es keine anderen Belege als deren Erinnerung gibt. So wird durch die ikonografische Fixierung individueller Erinnerungsbilder ein Geschichtsbild festgeschrieben, anstatt dessen Konstruktionscharakter offen zu legen.

Die Erinnerung von Zeitzeugen, zumal von Opfern wie etwa den nach dem 17. Juni inhaftierten Arbeitern, kritisch zu hinterfragen und Irrtümer sichtbar zu machen, ist immer auch ein ethisches Problem. Wenn aber interviewerprobte Politiker ähnlich zuvorkommend behandelt werden wie medienunerfahrene Bürger, resultiert daraus ein harmonisch-geglättetes Geschichtsbild wie in Deutschlandspiel. Zwar werden Zweifel und Kritik am raschen Vereinigungsprozess von Margaret Thatcher und François Mitterand sowie von sowjetischen Generälen in Spielszenen und in Interviews thematisiert, aber der Dissens bleibt immer ein historischer, kein historiografischer. Die Wahl des Zeitraums, von den Feiern zum vierzigsten Jahrestag der DDR als Anfang vom Ende bis zu ihrem tatsächlichen Ende am 3. Oktober 1990 zeigt den Prozess als eine einzige Niederlage ostdeutscher Politiker und als grandiosen Sieg des amtierenden Bundeskanzlers. Damit unterstützt Deutschlandspiel Helmut Kohls Arbeit an der eigenen Hagiografie. Zwar zeigt der Film Archivbilder seiner Rede mit dem Versprechen von »blühenden Landschaften« im Osten, aber eine Nachfrage an den Exkanzler, wie es sich denn damit zehn Jahre nach der Vereinigung verhalte, erfolgt nicht. In der Konzentration auf die Krise und den Untergang der DDR ist die Gegenwart als leitendes Erkenntnisinteresse an der Vergangenheit abwesend. So lässt sich die Entwicklung als gradlinige Erfolgsgeschichte erzählen. Andere Erzählungen, die »Erfolg« anders definieren, etwa diejenigen ostdeutscher Bürgerrechtler, welche die Revolution angestoßen haben, finden im von oben definierten Erinnerungsmodell dieses Films keinen Platz.

\section{Die DDR als grauer beziehungsweise bräunlich-pastellfarbener Osten}

Die Rekonstruktion der DDR in den Dokudramen entspricht generell dem Bild des grauen, tristen Ostens, der von Mangel, Unterdrückung und Propaganda geprägt ist. Set-Design und

\footnotetext{
${ }^{27}$ Roland Barthes: Historie und ihr Diskurs [frz. 1967], in: Alternative, Jg. 11 (1968), S. 171-180, hier S. 175.

${ }^{28}$ Tobias Ebbrecht: Sekundäre Erinnerungsbilder. Visuelle Stereotypenbildung in Filmen über Holocaust und Nationalsozialismus seit den 1990er Jahren, in: Christian Hißnauer/Andreas Jahn-Sudmann (Hg.): Medien - Zeit - Zeichen. Beiträge des 19. FFK, Marburg 2006, S. 37-44.
} 
Lichtsetzung bevorzugen ausgeblichene, bräunlich-pastellfarbene Töne. Dabei wird viel Wert auf die Rekonstruktion der historischen Kulissen, auf Originalschauplätze und authentische Kostüme und Objekte gelegt: Die Spielszenen der Dokudramen meinen es ernst mit ihrem »konkretistischen Illusionsnaturalismus $\ll^{29}$, der sowohl »Achtung Geschichte! « als auch $»$ Osten $=$ generalisierte Tristesse $«$ signalisiert. Die Straßen in Ostberlin sind zumeist menschenleer, wenn nicht von Polizei und/oder Militär bevölkert. Alltag ist dort die Ausnahme. In dem ästhetisierten Pastellbraun steckt auch ein Teil Faszination »von der Schönheit, die die Mangelwirtschaft der DDR produziert hat«, wie sie Matthias Dell dem Film Das Leben der Anderen (Florian Henckel von Donnersmarck, 2006) vorwirft. ${ }^{30}$ Anstatt solch »berückend triste[n] Minimalismus« zu zelebrieren, solle dieser besser zum Motiv der Auseinandersetzung werden. Die Dokudramen nehmen den »real existierenden Sozialismus« ausschließlich mit den Mitteln des Ausstattungskinos wahr, der nach der Formel: visuelle Klischees der Macht (Stacheldraht, Uniformen, Sichtagitation, Politikerporträts) plus Symbole des Alltags (Gummibäume, Marmorjeans, Gurkengläser, Blümchentapeten, Trabis) eine »potemkinsche Film-DDR ${ }^{31}{ }^{\text {schafft. }}$

Der Osten fungiert als Negativfolie des Westens. Allerdings bleibt die Bundesrepublik zumeist eine Chiffre für den besseren Teil und das bessere Leben, ohne dass eine Auseinandersetzung mit dieser erfolgt. Ein einziger Film verlässt dieses Muster: Die MauerBerlin '61 thematisiert Westarroganz ebenso wie Probleme von Ostdeutschen, sich in Westberlin zurechtzufinden. Der grauen DDR steht hier eine grelle BRD gegenüber, in der Sensationslust, Profitgier und Egoismus herrschen. Der Katalog der westkritischen Elemente hätte DEFA-Propagandafilmen der 1950er Jahre wie Slátan Dudows Der Hauptmann von Köln (1956) zur Ehre gereicht: Die wohlhabende Freundin des wider Willen im Westen gebliebenen Ehepaars weigert sich trotz Besitzes einer eigenen Villa, die in Not geratenen Bekannten bei sich aufzunehmen. Dafür können sie im Möbelgeschäft ihres Ehemannes übernachten, was dieser auch nur mit Hintergedanken angeboten hat: Seinem Werben gibt die Flüchtige schließlich nach, um den Rechtsanwalt bezahlen zu können, der ihren Sohn in den Westen holen soll. Das Treffen auf einem Golfplatz mit einem zynischen, Champagner trinkenden Juristen ist die kondensierte Karikatur antiwestlicher Feindbilder (Abb. 7).

\footnotetext{
${ }^{29}$ Gertrud Koch: Nachstellungen - Film und historischer Moment, in: Eva Hohenberger/Judith Keilbach (Hg.): Die Gegenwart der Vergangenheit. Dokumentarfilm, Fernsehen und Geschichte, Berlin 2003, S. 216-229, hier S. 226.

${ }^{30}$ Matthias Dell: Damaligenallee, in: Frankfurter Rundschau vom 31. März 2006, S. 15.

${ }^{31}$ Schmidt: Die DDR im Film, a. a. O.
} 
Schließlich finden sich die Eltern in der Enge eines überfüllten Notaufnahmelagers wieder und müssen erfahren, dass sie auf sich alleine gestellt sind; sie scheitern letzten Endes an den politischen Verhältnissen.

Abb. 7 Kondensierte Karikatur antiwestlicher Feindbilder: In Die Mauer - Berlin '61 empfängt der Anwalt die DDR-Flüchtlinge auf dem Golfplatz

\section{Bild- als Vergangenheitspolitik}

Stehen alle Filme im Rahmen des Systemkonfliktes unter dem Vorzeichen einer ideologischen Auseinandersetzung, so wird diese jedoch nicht politisch ausgetragen, sondern ist auf charakteristische Weise abwesend: In Die Mauer - Berlin '61 geraten die Hauptpersonen in Konflikt mit dem System, weil der Kupferdrahtdiebstahl des von Heino Ferch gespielten Arbeiters aufgeflogen ist und sich das Ehepaar aus Angst vor der Polizei nicht zurück in den Ostteil der Stadt traut. Die Verweigerung des Sohnes, sich für die SEDPropaganda missbrauchen zu lassen, ist eher ein körperlich-instinktiver als ein reflektierter Widerstand: Während der Brandrede, die er als Jungpionier gegen die republikflüchtigen »Rabeneltern« halten soll, äußert sich seine emotionale Krise physisch durch heftiges Nasenbluten. Die Szene zeigt, wie die ideologische Auseinandersetzung auf symbolischer Ebene über Farb- und Machtsymbolik geführt wird: Vor einer roten Thälmann-Fahne rinnt dem Jungen beim Zeremoniell das Blut aus der Nase und befleckt das weiße Ehrenhemd und das blaue Halstuch (Abb. 8). Ohne wie in Der Tunnel qualvoll lange Sterbeszenen an der Mauer zu inszenieren, zeigt der Film so die SED-Diktatur als blutbefleckt. Ihre Opfer sind unschuldige Kinder und »die Familie«, was in den Dokudramen über die DDR auf die Bevölkerung insgesamt ausgeweitet wird. Die Motivation der »Täter« wird generell nicht befragt. Die einzige Ausnahme stellt hierbei die oben erwähnte MDR-Produktion Tage des Sturms dar, in der SED-Funktionäre die gewaltsame Unterdrückung des Volksaufstandes 1953 mit ihrer biografischen Leidenserfahrung als Arbeiter in der NS-Diktatur rechtfertigen und als NS-Opfer zu Tätern werden.

Abb. 8 In Die Mauer - Berlin '61 wehrt sich der Körper des jugendlichen Protagonisten durch heftiges Nasenbluten, das die DDR als blutbefleckte Diktatur zeigt, gegen die ideologische Instrumentalisierung

Generell aber erfolgt die Thematisierung der Geschichte, die zur deutschen Zweistaatlichkeit geführt hat, nur im Subtext oder durch Randbemerkungen und mit Hilfe von Nebenfiguren. Der Umgang mit der Zeitgeschichte ist aber keinesfalls nebensächlich, sondern stellt ein wichtiges Erzählschema zur Integration der ehemaligen DDR-Bevölkerung in die bundesdeutsche Nachwendeordnung dar. 
In Zwei Tage Hoffnung rührt die Spaltung der Familie zwischen dem in Westberlin für den RIAS arbeitenden Sohn und den anderen sich in Ostberlin am Aufbau des Sozialismus beteiligenden Mitgliedern von einer Begebenheit im Krieg her. Nach den im wörtlichen Sinn schlagenden Argumenten, die den Vater die Brutalität des SED-Regimes am eigenen Leib haben erfahren lassen, erfolgt auf dem Krankenbett die tränenreiche Aussöhnung. Der Vater erklärt dem »verlorenen Sohn«, dass er schon immer gewusst habe, dass dieser nicht am Tod des kleinen Bruders in den letzten Kriegstagen schuld gewesen sei und bestätigt dessen in einem vorherigen Streit geäußerten Vorwurf: »Die Nazis ham’ ihn nicht umgebracht, das waren die Russen!« Sobald es um die NS-Zeit geht, setzt sich das Bedürfnis nach der »harmonischen Leinwand $\ll^{32}$ durch. Interessant ist, wie Zwei Tage Hoffnung den antifaschistischen Gründungsmythos der DDR delegitimiert und gegen das System wendet: Am Ende des Films wird der Kollege des in Ostberlin arbeitenden Bruders verhaftet, es handelt sich um Viktor, den Informanten des RIAS. Beim Anlegen der Handschellen wird eine eintätowierte Nummer auf seinem Arm erkennbar, betont durch eine Großaufnahme (Abb. 9-10).

Abb. 9-10 Delegitimierung des antifaschistischen Gründungsmythos der DDR anhand der eintätowierten Nummer bei der Verhaftung des Informanten Viktor in Zwei Tage Hoffnung

Damit rekurriert Zwei Tage Hoffnung auf ein »Superzeichen« der Erinnerung an die Judenvernichtung. Es kommt zu einer »Ablösung ästhetischer und narrativer Formen aus dem Kontext des Holocaust-Films, die auf [...] das Schicksal der deutschen Bevölkerung übertragen werden. ${ }^{33}$ So wird die Konstruktion der Deutschen als Opfer des Nationalsozialismus, wie sie sich durch die Dokudramen über das »Dritte Reich« zieht, auf die DDR-Bevölkerung übertragen. Implizit schwingt die Botschaft $» \mathrm{DDR}=\mathrm{KZ} \ll$ mit, wie sie ab 1961 auf die Berliner Mauer geschrieben wurde und in westdeutschen Mauerfilmen dann weltweit zu sehen war. ${ }^{34}$ In diesem Kontext ist auch »Viktor« als Deckname des Informanten kein Zufall: im Geiste von Casablanca (Michael Curtiz, 1942) arbeiten die Antifaschisten für Westberlin.

\footnotetext{
32 Sonja M. Schultz: Die harmonische Leinwand. Filmische Stereotypen bei der Darstellung von Nationalsozialismus und Holocaust in aktuellen deutschen Produktionen, in: Erika Richter/Ralf Schenk/Claus Löser (Hg.): apropos: Film 2005 - Das Jahrbuch der DEFA-Stiftung, Berlin 2005, S. 76-88.

${ }^{33}$ Ebbrecht: Sekundäre Erinnerungsbilder, a. a. O., S. 42.

${ }^{34}$ Vgl. Steinle: Vom Feindbild zum Fremdbild, a. a. O., S. 289.
} 
Die NS-Vergangenheit ist auch in Der Tunnel durch eine Nebenerzählung präsent: Der Student Fred von Klausnitz beteiligt sich am Tunnelbau, um seine alleinstehende Mutter in den Westen zu holen. Als Adelige hat sie einen schweren Stand im Sozialismus. Im Laufe der Handlung stellt sich heraus, dass ihr Mann am militärischen Widerstand gegen Hitler beteiligt war und deswegen sterben musste. Nachdem die Stasi von den Fluchtplänen erfahren hat und die alte Frau abholen will, entzieht sie sich dem Verhör, indem sie sich symbolträchtig mit der Pistole ihres Mannes erschießt. Damit steht hier die DDR ebenfalls in Täterkontinuität zum »Dritten Reich«, und auch in diesem Film wird die Gegenwart der Vergangenheit über die entlastende Personalunion von Opfer und Widerstandskämpfer thematisiert. In den Dokudramen über die DDR war Opa (und Oma) kein Nazi. ${ }^{35}$

Das Erzählmotiv von der DDR als Fortsetzung der NS-Diktatur unter roten Vorzeichen schließt an antitotalitaristische Theoriebildung an, delegitimiert den moralisch aufgeladenen antifaschistischen Gründungsmythos der DDR und legitimiert wiederum deren schnelle Abwicklung nach 1989 unter den Vorzeichen des ökonomisch orientierten westdeutschen Gründungsmythos von Wirtschaftswunder und D-Mark. Die geschichtspolitische Gleichsetzung der Systeme auf struktureller Ebene konterkarieren die Filme durch die Adaption von Opferzeichen aus den Holocaust-Erzählungen zur Markierung der DDR-Bürger als Opfer des Systems. Mit der Selbstbefreiung, zu der die Deutschen im Nationalsozialismus nicht fähig waren, weisen die Dokudramen der ehemaligen DDR-Bevölkerung einen bevorrechtigten Platz in der Geschichte zu, was unter anderem auch als Gegengewicht zur westdeutschen diskursiven und ökonomischen Dominanz betrachtet werden kann.

\section{Abspann: Der vergessenen Farbfilm}

Das DDR-Bild der Dokudramen des ersten Jahrzents im neuen Jahrtausend steht in Kontinuität narrativer und ästhetischer Stereotype vom Kalten Krieg, die durch die Konzentration auf Krisenmomente und den Rekurs auf Genremuster befördert werden. DDRBürger sind in erster Linie Opfer - selbst wenn sie überzeugte Kommunisten sind wie in Zwei Tage Hoffnung. Damit bieten die Filme ein Narrativ an, das mit der Ex-post-Konstruktion einer großen Leidensgemeinschaft anschlussfähig ist an die Erinnerung an den Nationalsozialismus und kompatibel mit der westdeutsch dominierten Erinnerungskultur. Vertreter des Systems sind fast ausschließlich stilisierte Klischee-Bösewichte, Stasi-Schergen,

\footnotetext{
${ }^{35}$ Vgl. Harald Welzer/Sabine Moller/Karoline Tschuggnall: »Opa war kein Nazi«. Nationalsozialismus und Holocaust im Familiengedächtnis, Frankfurt am Main 2002.
} 
die zum Teil ohne Kostümwechsel in vor 1945 angesiedelten Dokudramen mitspielen könnten. Den wenigsten Kommunisten gestattet das Drehbuch, »aus ehrlicher Überzeugung an der sozialistischen Idee festgehalten zu haben $\ll^{36}$, wie die Ausnahmehauptfigur in Das Wunder von Berlin, die eine Entwicklung vom Punk zum überzeugten NVA-Soldaten durchmacht. Der Preis für subjektive Aufrichtigkeit in der Figurenkonstruktion ist naiver Idealismus oder einfach nur Naivität, wie sie die menschlich herzlichen aber mental einfach strukturierten Wodka trinkenden Linken in der ebenfalls von Roland S. Richter gedrehten Heteotopie Die Grenze charakterisiert.

Entgegen der Tendenz zum kitschigen Happyend vieler Event-Movies stehen auch traurigtragische Enden wie in Zwei Tage Hoffnungoder Die Mauer - Berlin '61. Letzterer stellt insofern eine Ausnahme dar, indem er eine westkritische Perspektive einnimmt und schmerzhafte Fremdheitserfahrung thematisiert. Die Mauer - Berlin '6lbuchstabiert Freiheit auch ökonomisch - und entsprechend bleiben die unfreiwilligen Flüchtlinge Gefangene ihrer beschränkten Mittel. Damit fällt der Film aus dem Rahmen westdeutscher Heilserzählungen und verweigert mit dem gescheiterten Fluchtversuch des Sohnes nicht nur ein Happyend, sondern auch eine retrospektive Tröstung, wenn der abschließende Sprecherkommentar verkündet: »Die Kulkes warteten, sie warteten 28 Jahre ... und als sie sich wieder trafen, waren sie sich fremd.«

Komplett abwesend in allen Filmen ist die Sphäre des Alltags in seiner banalen Form, das heißt als gelebtes und nicht nur erlittenes Leben. ${ }^{37}$ In dieser charakteristischen Auslassung erklärt sich die in anderen Medien zelebrierte ungebrochene Faszinationskraft der DDRBilder und Produktwelt, in der weniger kurze Krisenmomente als vielmehr langfristige Erinnerungen stecken. Dass die Faszination sich gerade nicht an Herrschafts- und Machtsymbolen, sondern am Sandmännchen, am Kobold Pittiplatsch oder an der Ente Schnatterinchen festmacht, zeugt weniger von einem Sieg des SED-Systems in der letzten Schlacht des Kalten Krieges ${ }^{38}$, als vielmehr von biografisch-erinnerungskulturellem Eigensinn. Die thematische und inhaltliche Engführung der DDR in Dokudramen bietet einerseits Anknüpfungspunkte für ostdeutsche Erinnerung und gesamtdeutsche Integration,

\footnotetext{
${ }^{36}$ Ralf Schenk: Die DDR im deutschen Film nach 1989, in: Aus Politik und Zeitgeschichte, Jg. 55 (2005), Bd. 44, S. 31-38, hier S. 38.

${ }^{37}$ Vgl. Schmidt: Die DDR im Film, a. a. O.

${ }^{38}$ So bei Stefan Wolle: Die Welt der verlorenen Bilder. Die DDR im visuellen Gedächtnis, in: Gerhard Paul (Hg.): Visual History. Ein Studienbuch, Göttingen 2006, S. 333-352, hier S. 333 f.
} 
andererseits aber bleiben Leerstellen, so dass der Abspann von Sonnenallee (Leander Haußmann, 1999) als Echo nachhallt: In diesem besingt Nina Hagen laut klagend den vergessenen Farbfilm, was sich zur Entstehungszeit des Liedes 1974 ironisch gegen die aufgezwungene Enge und Langeweile in der DDR richtete. Im Nachwende-Deutschland zielt der Einsatz des Liedes auch gegen die schwarz-weiße oder in Graustufen und Pastellfarben wahrgenommene Darstellung der DDR ${ }^{39}$, die ungeachtet aller visueller Opulenz, aller Stars und Special Effects die Dokudramen kennzeichnet.

Für die relative Abwesenheit von Dokudramen über die DDR im gegenwärtigen deutschen Fernsehen mögen nicht nur die Verrentung von Guido Knopp und das Verschwinden des Firmennamens teamWorx ${ }^{40}$ jeweils 2013 verantwortlich sein. In den letzten Jahren sind eine Reihe von Filmen mit Geschichten aus der DDR entstanden, die das Leben in dieser jenseits der Krisendaten wahrnehmen: Zu nennen sind vor allem Barbara (2012) von Christian Petzold und Westen (2013) von Christian Schwochow, die beachtliche Zuschauerzahlen hatten und auch international auf Aufmerksamkeit stießen. Deren Erfolg ließe sich auch als Anzeichen dafür interpretieren, dass sich ein Vierteljahrhundert nach dem Verschwinden der DDR deren (Re-)Konstruktionen auf Leinwand und Bildschirm ausdifferenzieren und komplexere Darstellungen auch ein größeres Publikum erreichen. Ein Wunder braucht die DDR dafür nicht einmal...

\footnotetext{
39 Vgl. Thomas Lindenberger: Zeitgeschichte am Schneidetisch. Zur Historisierung der DDR in deutschen Spielfilmen, in: Paul (Hg.): Visual History, a. a. O., S. 353-372, hier S. 358.

${ }^{40} 2013$ wurde im Rahmen einer Umstrukturierung teamWorx mit anderen UFA-Tochtergesellschaften zur neuen Firma UFA Fiction zusammengelegt.
} 